\title{
Cognitive bias in the clinical decision making of doctors
}

\author{
Authors: Thomas O'Hagan, ${ }^{\mathrm{A}}$ John Fennell, ${ }^{\mathrm{B}}$ Kean Tan, ${ }^{\mathrm{A}}$ Davina Ding ${ }^{\mathrm{A}}$ and Iolo Thomas-Jones ${ }^{\mathrm{A}}$
}

\section{Aims}

To investigate whether medics are susceptible to one of the most widely studied cognitive biases, anchoring; an error in thought processing where decisions are strongly influenced by previously presented information.

\section{Methods}

Referrals to the acute medical unit (AMU) at the Bristol Royal Infirmary were retrospectively recorded over a 16-day period. Referrals were categorised from the information available on the medical take sheet into potential diagnosis, symptoms alone, and signs, investigations and patient observations. Patients were followed up in order to ascertain the diagnosis at discharge. The results were analysed to investigate whether the type of information given to a doctor prior to reviewing a patient would affect their initial diagnosis. Results were reviewed for both junior doctors and consultants at the post-take ward round.

\section{Results}

Overall, junior doctors were more likely to arrive at the correct diagnosis when no potential diagnosis was given on the medical take list ( $p=0.025$ ), but for consultants there was no significant difference $(p=0.11)$. However, both junior doctors $(p=0.017)$ and consultants ( $p=0.025)$ were significantly more likely to reach a correct diagnosis when provided with patient signs, investigation results or observations, rather than being given a potential diagnosis. There was no significant difference for junior doctors $(p=0.12)$, or consultants $(p=0.18)$ when comparing being given purely patient symptoms to a possible diagnosis.

\section{Conclusion}

Taken together, our findings suggest that the gold standard for presenting information to doctors when patients arrive at the AMU is with examination or investigation findings, rather than with a possible diagnosis (which the doctor can be strongly anchored to), or via symptoms alone (not enough information). This represents a cost-neutral intervention to improve the efficiency junior doctors correctly diagnose patients.

\section{Conflict of interest statement}

All authors declare that there is no conflict of interest.

Authors: ${ }^{\text {AF }}$ aculty of Health Sciences; ${ }^{B}$ School of Experimental Psychology, University of Bristol, Bristol, UK 